\title{
Soluble Fibrin Degradation Products Potentiate Tissue Plasminogen Activator-induced Fibrinogen Proteolysis
}

Jeffrey I. Weitz, Beverly Leslie, and Jeffrey Ginsberg

Department of Medicine, McMaster University, and Hamilton Civic Hospitals Research Centre, Hamilton, Ontario L8V IC3 Canada

\begin{abstract}
Despite its affinity for fibrin, tissue plasminogen activator (tPA) administration causes systemic fibrinogenolysis. To investigate the mechanism, t-PA was incubated with plasma in the presence or absence of a fibrin clot, and the extent of fibrinogenolysis was determined by measuring $B \beta 1-42$. In the presence of fibrin, there is a 21-fold increase in $B \beta 1-42$ levels. The potentiation of fibrinogenolysis in the presence of fibrin is mediated by soluble fibrin degradation products because $(a)$ the extent of t-PA induced fibrinogenolysis and clot lysis are directly related, (b) once clot lysis has been initiated, fibrinogenolysis continues even after the clot is removed, and (c) lysates of cross-linked fibrin clots potentiate t-PA-mediated fibrinogenolysis. Fibrin degradation products stimulate fibrinogenolysis by binding t-PA and plasminogen because $\sim 70 \%$ of the labeled material in the clot lysates binds to both t-PA- and plasminogen-Sepharose, and only the bound fractions have potentiating activity. The binding site for t-PA and plasminogen is on the $\mathbf{E}$ domain because characterization of the potentiating fragments using gel filtration followed by PAGE and immunoblotting indicates that the major species is (DD)E complex, whereas minor components include high-molecular weight derivatives containing the (DD)E complex and fragment $E$. In contrast, D-dimer is the predominant species found in the fractions that do not bind to the adsorbants, and it has no potentiating activity. Thus, soluble products of $t$-PA-induced lysis of cross-linked fibrin potentiate t-PA-mediated fibrinogenolysis by providing a surface for t-PA and plasminogen binding thereby promoting plasmin generation. The occurrence of this phenomenon after therapeutic thrombolysis may explain the limited clot selectivity of t-PA. (J. Clin. Invest. 1991.87:10821090.) Key words: thrombolysis - (DD)E complex • plasmin generation
\end{abstract}

\section{Introduction}

Tissue-type plasminogen activator (t-PA) ${ }^{1}$ converts plasminogen to plasmin, thereby initiating fibrinolysis (1). The property that distinguishes t-PA from urokinase, the other major endoge-

Address correspondence to Dr. Jeffrey Weitz, Henderson General Hospital, 711 Concession St., Hamilton, Ontario L8V 1C3 Canada.

Received for publication 20 August 1990 and in revised form 15 October 1990

1. Abbreviations used in this paper: DD, D-dimer; rt-PA, recombinant tissue-type plasminogen activator; t-PA, tissue-type plasminogen activator.

J. Clin. Invest.

(c) The American Society for Clinical Investigation, Inc. 0021-9738/91/03/1082/09 \$2.00

Volume 87, March 1991, 1082-1090 nous plasminogen activator, is its relative fibrin selectivity. Both t-PA and plasminogen bind to fibrin (1-4) where they undergo conformational changes $(5,6)$ which promote their interaction and enhance plasmin generation on the clot surface $(7,8)$, where it is relatively protected from inactivation by $\alpha_{2}$ antiplasmin $(1,4)$. Through this mechanism, t-PA has the potential to produce clot lysis without inducing a systemic lytic state.

The clot-selective properties of t-PA provided the major impetus for the development of t-PA as a therapeutic agent. With the successful cloning and expression of t-PA cDNA (9), amounts of recombinant t-PA (rt-PA) sufficient for clinical studies have recently become available. Although rt-PA is an extremely effective thrombolytic agent, its use in patients with acute myocardial infarction (10-15) and venous thromboembolic disease (16-19) results in significant fibrinogen proteolysis, albeit to a lesser extent than that produced by streptokinase (12-15). Thus, large clinical studies have demonstrated that in pharmacologically effective doses, rt-PA is not fibrin-specific.

There are two potential reasons why rt-PA administration is associated with systemic fibrinogenolysis despite the in vitro evidence that t-PA is clot-specific. One is based on theoretical calculations of the reported kinetic parameters for the interaction between plasminogen and t-PA. Using computer-assisted simulation, these models predicted that thrombolytic doses of rt-PA would produce systemic fibrinogenolysis (20, 21). Another potential explanation for the limited clot selectivity of rt-PA is that products of thrombolysis augment fibrinogen proteolysis. This concept is supported by the results of in vitro studies demonstrating that fibrin fragments accelerate plasminogen activation by t-PA in buffer systems (22-28). In this investigation, we performed studies in a plasma system $(a)$ to determine whether soluble degradation products of crosslinked fibrin can potentiate rt-PA induced fibrinogenolysis, and $(b)$ to explore the mechanism of potentiation.

\section{Methods}

Reagents. Predominantly single-chain human rt-PA (lot K9051A6) was obtained from Genentech, Inc., San Francisco, CA. Fragment E was purchased from Diagnostica Stago, Asnieres, France. Plasminogen with an amino-terminal glutamic acid residue (glu-plasminogen) and mouse monoclonal antibody against human t-PA (PAM-2) were purchased from American Diagnostica, New York, NY. A mouse monoclonal antibody against D-dimer (DD), designated 8D3, was generously provided by Dr. D. Collen, University of Leuven, Belgium. A horseradish peroxidase-conjugated monospecific goat IgG against human fibrinogen was obtained from Cooper Biomedical, Inc., Malvern, PA. Preliminary immunoblotting studies with this antibody indicated that it recognizes intact fibrinogen and the plasmin-derived fragments $\mathrm{X}, \mathrm{Y}$, and $\mathrm{D}$. It does not, however, react with fragment $\mathrm{E}$. A monospecific rabbit antibody against fragment $E$ was purchased from Diagnostica Stago. Immunoblotting studies using this antibody demonstrated that it recognizes intact fibrinogen, and fragments $\mathrm{X}, \mathrm{Y}$, and $\mathrm{E}$. It does not react with fragment $\mathrm{D}$ or $\mathrm{DD}$. 
Preparation of ${ }^{125}$ I-labeled fibrinogen. Fibrinogen was precipitated from barium sulfate-adsorbed plasma with $2 \mathrm{M} \beta$-alanine as described in detail elsewhere (29). The isolated fibrinogen was then trace labeled with ${ }^{125} \mathrm{I}(30)$ to a specific activity of $100 \pm 5 \mu \mathrm{Ci} / \mathrm{mg}$.

Preparation of ${ }^{125}$ I-labeled cross-linked fibrin clots. Blood was collected from the antecubital veins of healthy volunteers into plastic syringes prefilled with $1 / 10 \mathrm{vol}$ of $3.8 \%$ trisodium citrate. After thorough mixing with the anticoagulant, the red cells were sedimented by centrifugation at $1,700 \mathrm{~g}$ for $15 \mathrm{~min}$ at $4^{\circ} \mathrm{C}$. The harvested plasma was supplemented with ${ }^{125} \mathrm{I}$-labeled fibrinogen $(\sim 120,000 \mathrm{cpm} / \mathrm{ml})$ and $500-\mu \mathrm{I}$ aliquots were then transferred to polypropylene eppendorf tubes. Labeled cross-linked fibrin clots were formed around wire coils by the addition of $\mathrm{CaCl}_{2}$ (final concentration, $25 \mathrm{mM}$ ). For some experiments, ${ }^{125} \mathrm{I}$-labeled fibrin clots of varying sizes were prepared by the recalcification of plasma in volumes ranging from $250 \mu \mathrm{l}$ to $1 \mathrm{ml}$. In all cases however, the clots were aged for $60 \mathrm{~min}$ at $37^{\circ} \mathrm{C}$ with constant agitation, and then washed three times with $1-\mathrm{ml}$ aliquots of $0.1 \mathrm{M}$ $\mathrm{NaCl}$ buffered with $0.05 \mathrm{M}$ Tris- $\mathrm{HCl}, \mathrm{pH} 7.4$ (TBS) over the course of $30 \mathrm{~min}$. The washed clots were then counted for radioactivity for $1 \mathrm{~min}$ using a Clinigamma counter (LKB Instruments, Inc., Gaithersburg, MD).

Clots formed in this fashion are cross-linked because they remain intact after $24 \mathrm{~h}$ incubation in $2 \%$ acetic acid. Further, SDS-PAGE analysis under reducing conditions of clots solubilized in SDS as described by Francis et al. (31) demonstrates bands corresponding to the $\beta$, gamma-gamma dimers, and $\alpha$-polymer chains (data not shown). Non-cross-linked $\alpha$ - or gamma-chains are not present, thus indicating virtually complete cross-linking.

Quantification of rt-PA-induced fibrin(ogen)olysis in the presence or absence of ${ }^{125}$ I-labeled fibrin clots. rt-PA (at concentrations ranging from 0.125 to $2.0 \mu \mathrm{g} / \mathrm{ml}$ ) was incubated with $500-\mu \mathrm{l}$ aliquots of fresh citrated plasma for $60 \mathrm{~min}$ at $37^{\circ} \mathrm{C}$ in the presence or absence of ${ }^{125} \mathrm{I}-\mathrm{la}$ beled fibrin clots, and clot lysis and fibrinogenolysis were then monitored as follows. (a) Clot lysis. The extent of rt-PA-induced clot lysis was quantified $(i)$ by monitoring the time-course of release of ${ }^{125} \mathrm{I}$-labeled fibrin degradation products, and (ii) by removing the fibrin clots from the rt-PA containing plasma, and after washing three times with $500-\mu l$ aliquots of TBS, counting the residual radioactivity for $1 \mathrm{~min}$. The difference between the radioactivity originally incorporated in the clot and the residual radioactivity was then expressed as a percentage of the initial radioactivity. (b) Fibrinogenolysis. The extent of rt-PA-induced fibrinogenolysis was determined by monitoring plasma levels of B $\beta 1-42$. At intervals, $100-\mu \mathrm{l}$ aliquots of plasma were removed, and unreacted fibrinogen was precipitated by the addition of $300 \mu \mathrm{l}$ of chilled ethanol followed by centrifugation at $15,000 \mathrm{~g}$ for $5 \mathrm{~min}$. The ethanol supernatants were then evaporated to dryness in a Speed-Vac Concentrator (Savant Instruments, Inc., Farmingdale, NY), reconstituted to original volume with distilled water, and assayed for $\mathrm{B} \beta 1-42$. In some experiments, immunoblot analysis was used to directly visualize the fibrinogen degradation products. For these studies, $20-\mu 1$ aliquots were removed at intervals, diluted in an equal volume of $60 \mathrm{mM}$ Tris- $\mathrm{HCl}$ containing $2 \%$ SDS, $5 \%$ glycerol, and $0.001 \%$ bromophenol blue, heated at $100^{\circ} \mathrm{C}$ for $5 \mathrm{~min}$, and then stored at $-70^{\circ} \mathrm{C}$ until analyzed as described below.

Radioimmunoassay of $B \beta 1-42$. The fibrinogen-derived fragment B $\beta 1-42$ was measured by radioimmunoassay as previously described using a specific antibody that does not cross-react with fibrinopeptide $B$ or $\mathrm{B} \beta 15-42$ (32).

Preparation of clot lysates. To prepare clot lysates, ${ }^{125} \mathrm{I}$-labeled cross-linked fibrin clots were incubated for $60 \mathrm{~min}$ at $37^{\circ} \mathrm{C}$ in $500-\mu 1$ aliquots of TBS containing $4 \mu \mathrm{g} / \mathrm{ml} \mathrm{rt}-\mathrm{PA}$ and $0.02 \%$ Tween 80 . At the end of the incubation period, the residual clots were removed, and the clot lysates were then pooled and immunodepleted of rt-PA by affinity chromatography. A mouse monoclonal IgG against the kringle-1 region (33) of human t-PA (PAM-2, American Diagnostica) was coupled to cyanogen bromide activated $\mathrm{CH}$ Sepharose 4B (Pharmacia Fine Chemicals, Piscataway, NJ) at a concentration of $20 \mathrm{mg} / \mathrm{ml}$. The clot lysates $(10 \mathrm{ml})$ and anti-t-PA IgG coupled to $\mathrm{CH}$ Sepharose 4B $(750 \mu \mathrm{l}$ of a $50 \%$ suspension) were mixed in a tube and agitated for $1 \mathrm{~h}$ at $23^{\circ} \mathrm{C}$. After centrifugation at $10,000 \mathrm{~g}$ for $5 \mathrm{~min}$, the supernatants were carefully removed, and the immunodepletion procedure was then repeated for two additional 1-h cycles. The final material contained $<50 \mathrm{ng}$ of t-PA as measured antigenically using an enzyme-linked immunosorbent assay kit from American Diagnostica. The lysates were then concentrated 10-fold using an ultrafiltration cell (series 8050, Amicon Division, W. R. Grace \& Co., Danvers, MA) fitted with a 5,000-mol wt cut-off membrane (YM5 disc membrane, Amicon), counted for radioactivity for $1 \mathrm{~min}$, and stored in aliquots at $-70^{\circ} \mathrm{C}$.

The effect of clot lysates on rt-PA-mediated fibrinogenolysis. To determine whether soluble fibrin degradation products potentiate rtPA-mediated fibrinogenolysis, varying amounts of clot lysate or buffer control were incubated in $500-\mu \mathrm{l}$ aliquots of citrated plasma for $60 \mathrm{~min}$ at $37^{\circ} \mathrm{C}$ in the presence or absence of rt-PA (at a concentration of 1 or 2 $\mu \mathrm{g} / \mathrm{ml})$. At intervals, $100-\mu \mathrm{l}$ aliquots were removed, and the unreacted fibrinogen was precipitated with $300 \mu \mathrm{l}$ of chilled ethanol followed by centrifugation at $15,000 \mathrm{~g}$ for $5 \mathrm{~min}$. The ethanol supernatants were then evaporated to dryness, reconstituted to original volume with distilled water, and assayed for $\mathrm{B} \beta 1-42$.

Adsorption of ${ }^{125}$ I-labeled fibrin degradation products to plasminogen-Sepharose or rt-PA-Sepharose. Glu-plasminogen and rt-PA were each coupled to cyanogen bromide-activated $\mathrm{CH}$ Sepharose 4B (Pharmacia Fine Chemicals) at a concentrations of 5 and $1 \mathrm{mg} / \mathrm{ml}$, respectively. Lysates of ${ }^{125} \mathrm{I}$-labeled fibrin clots were then subjected to affinity chromatography at $23^{\circ} \mathrm{C}$ on columns $(5 \times 0.7 \mathrm{~cm})$ containing either glu-plasminogen or rt-PA coupled to $\mathrm{CH}$ Sepharose 4B. 1-ml fractions were collected and their radioactivity and absorbance at $280 \mathrm{~nm}$ were determined. After extensive washing of the columns with TBS, material bound to plasminogen-Sepharose was eluted with TBS containing $0.05 \mathrm{M}$ lysine, whereas that bound to rt-PA-Sepharose was eluted with $0.4 \mathrm{M} \mathrm{NaCl}$ buffered to $\mathrm{pH} 4.0$ with $0.1 \mathrm{M}$ ammonium acetate. Again, $1-\mathrm{ml}$ fractions were collected, and their radioactivity and absorbance at $280 \mathrm{~nm}$ were determined. After confirming the protein concentration using the method of Lowry (34), peak protein-containing fractions were pooled, concentrated using Centricon- 10 ultrafiltration cartridges (Amicon Division, W. R. Grace \& Co.), and then tested for their ability to potentiate rt-PA-induced fibrinogenolysis in plasma using the method described above.

Gel filtration of clot lysates. Crude lysates of ${ }^{125}$ I-labeled fibrin clots and material eluted from either the plasminogen-Sepharose or the rtPA-Sepharose columns were subjected to chromatography at $23^{\circ} \mathrm{C}$ on a column $(90 \times 1.6 \mathrm{~cm})$ of Sephacryl S-300 HR (Pharmacia Fine Chemicals) using TBS at a rate of $50 \mathrm{ml} / \mathrm{h}$. For preparative procedures, Dextran blue (Sigma Chemical Co., St. Louis, MO) was used to determine the void volume, and the column was calibrated with thyroglobulin, gamma-globulin, ovalbumin, myoglobulin, and cyanocobalamin with molecular weights of $670,000,158,000,44,000,17,000$, and 1,350 D, respectively. $2-\mathrm{ml}$ fractions were collected and their radioactivity and absorbance at $280 \mathrm{~nm}$ were measured. After confirming the protein concentration using the method of Lowry (34), peak-protein containing fractions were pooled, concentrated using Centricon-10 ultrafiltration cartridges (Amicon Division, W. R. Grace \& Co.), and then tested for their ability to potentiate rt-PA-induced fibrinogenolysis in plasma using the method described above.

$P A G E$ and immunoblot analysis of fibrin(ogen) degradation products. Plasma incubated with rt-PA in the presence or absence of fibrin clots, crude clot lysates, the fibrin degradation products that bound and those that did not bind to plasminogen-Sepharose or to rt-PA-Sepharose, and the peak protein-containing fractions recovered from the gel filtration column were characterized using a combination of PAGE and immunoblot analysis. Samples were diluted in an equal volume of $60 \mathrm{mM}$ Tris- $\mathrm{HCl}$ containing $0.001 \%$ bromphenol blue with or without $2 \%$ SDS and 5\% glycerol. Two electrophoretic systems were used: a $7.5 \%$ polyacrylamide slab gel ( $4 \%$ stacking gel) containing $0.1 \%$ SDS using a modified Laemmli discontinuous buffer system (35) under 
nonreducing conditions and a $7.5 \%$ polyacrylamide gel (4\% stacking gel) under nondisocciating conditions according to the method of Davis (36). The gels were either fixed in $40 \%$ methanol/10\% acetic acid and stained with Coomasie Blue, or the separated proteins were electrophoretically transferred onto nitrocellulose membranes. After blocking in $5 \%$ (wt/vol) fat-free milk diluted in $0.15 \mathrm{M} \mathrm{NaCl}$, the membranes were washed and incubated for $90 \mathrm{~min}$ with either a 1:3,000 dilution of horseradish peroxidase-conjugated goat anti-human fibrinogen IgG (Cooper Biomedical, Inc.), or a 1:1,000 dilution of rabbit antibody against Fragment E (Diagnostica Stago) or mouse monoclonal IgG against DD (8D3). In the case of the latter two antibodies, the washed membranes were incubated for $60 \mathrm{~min}$ with a 1:1,000 dilution of horseradish peroxidase-conjugated goat anti-rabbit or anti-mouse IgG (BioRad Laboratories, Inc., Richmond, CA), and all the membranes were then developed with chloronaphthol followed by hydrogen peroxide.

\section{Results}

Formation of ${ }^{125}$ I-labeled fibrin clots. The recalcification of $500-\mu \mathrm{l}$ aliquots of plasma containing ${ }^{125} \mathrm{I}$-labeled fibrinogen results in the formation of fibrin clots of standard size. $\sim 95 \%$ of the radiolabeled fibrinogen is incorporated into the clots so that the radioactivity is $56,943 \pm 1,180 \mathrm{cpm}$ (mean $\pm \mathrm{SD}$ ).

Increased rt-PA-mediated fibrinogenolysis in the presence of fibrin clots. To determine the influence of fibrin on rt-PAmediated fibrinogenolysis, plasma containing increasing concentrations of rt-PA was incubated in the presence or absence of an ${ }^{125} \mathrm{I}$-labeled cross-linked fibrin clot, and $\mathrm{B} \beta 1-42$ was measured as an index of fibrinogenolysis. As illustrated in Table I, in the absence of fibrin, rt-PA produces concentration-dependent generation of small amounts of $B \beta 1-42$ that represent $<1 \%$ of the total releasable $\mathrm{B} \beta 1-42$ (as calculated from the plasma fibrinogen concentration of $6.2 \mu \mathrm{M}$ ). In contrast, in the presence of fibrin there is $\sim 21$-fold increase in the amount of $B \beta 1-42$ produced by each concentration of rt-PA. This increase is not due to the release of trapped $B \beta 1-42$ from within the clots because $<10 \mathrm{nM} \mathrm{B} \beta 1-42$ is recovered when clots suspended in buffer are completely lysed by rt-PA (data not shown).

Table I. rt-PA-mediated BB1-42 Release in Citrated Plasma in the Presence and Absence of a Fibrin Clot

\begin{tabular}{ccc}
\hline & \multicolumn{2}{c}{$\mathrm{B} \beta 1-42$} \\
\cline { 2 - 3 } rt-PA & No clot & Clot \\
\hline$\mu g / m l$ & & $n M$ \\
& & \\
None & $0.6 \pm 0.1$ & $2.3 \pm 0.9$ \\
0.125 & $5.4 \pm 0.8$ & $113.3 \pm 10.6$ \\
0.250 & $13.9 \pm 2.6$ & $269.2 \pm 15.2$ \\
0.500 & $21.9 \pm 4.8$ & $393.5 \pm 21.3$ \\
1.000 & $56.3 \pm 9.8$ & $1786.3 \pm 31.2$ \\
2.000 & $91.2 \pm 10.1$ & $2243.3 \pm 38.9$ \\
& & \\
\hline
\end{tabular}

Citrated plasma was incubated with increasing concentrations of rtPA for $60 \mathrm{~min}$ at $37^{\circ} \mathrm{C}$ in the presence or absence of a fibrin clot. At the end of incubation period, unreacted fibrinogen was precipitated with ethanol, and the ethanol supernatants were assayed for B $\beta 1-42$. The results illustrated represent the mean $\pm S D$ of three separate experiments.

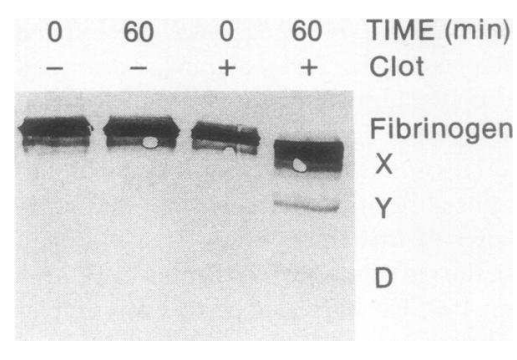

Figure 1. Immunoblot analysis of rt-PAinduced fibrinogenolysis in the presence or absence of a fibrin clot. Citrated plasma was incubated with $1 \mu \mathrm{g} / \mathrm{ml}$ of rt-PA for $60 \mathrm{~min}$ at $37^{\circ} \mathrm{C}$ in the presence or absence of a fibrin clot.

At the times indicated, aliquots of plasma were removed and subjected to PAGE and immunoblot analysis as described in Methods.

That the increase in $\mathrm{B} \beta 1-42$ levels in the presence of a clot reflects enhanced fibrinogen proteolysis is confirmed by the results of immunoblot analysis (Fig. 1). Marked fibrinogen proteolysis is produced by $1 \mu \mathrm{g} / \mathrm{ml}$ of rt-PA in the presence of a fibrin clot but not in its absence.

Relationship between rt-PA-induced clot lysis and fibrinogenolysis. ${ }^{125} \mathrm{I}$-labeled fibrin clots of different sizes were incubated for $60 \mathrm{~min}$ at $37^{\circ} \mathrm{C}$ in plasma containing $1 \mu \mathrm{g} / \mathrm{ml} \mathrm{rt}-\mathrm{PA}$, and clot lysis and fibrinogenolysis were quantified. This concentration of rt-PA was chosen because it represents a therapeutic level (37). To determine the extent of fibrinogenolysis, the amount of $B \beta 1-42$ generated was expressed as a percent of the total releasable $\mathrm{B} \beta 1-42$ (as calculated from the plasma fibrinogen concentration of $6.2 \mu \mathrm{M}$ ). As illustrated in Fig. 2, there is a linear relationship between the percent of $B \beta 1-42$ released, and the extent of clot lysis.

To investigate the mechanism for the relationship between clot lysis and fibrinogenolysis, ${ }^{125} \mathrm{I}$-labeled fibrin clots of a standard size were incubated for $60 \mathrm{~min}$ at $37^{\circ} \mathrm{C}$ in $500-\mu \mathrm{l}$ aliquots of plasma containing $1 \mu \mathrm{g} / \mathrm{ml} \mathrm{rt}$-PA. At varying intervals, the clots were removed, and the time-course of clot lysis was monitored by measuring the release of ${ }^{125} \mathrm{I}$-labeled fibrin degradation

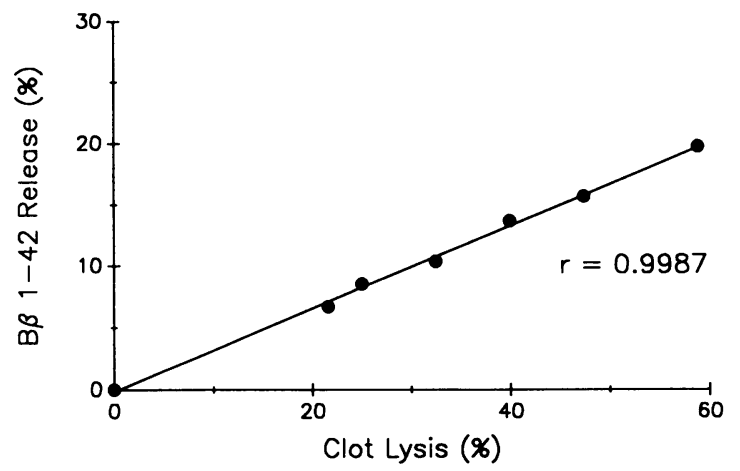

Figure 2. Relationship between the extent of $\mathrm{B} \beta 1-42$ release and the percentage of clot lysis. ${ }^{125} \mathrm{I}$-labeled fibrin clots of different sizes were incubated in plasma containing $1 \mu \mathrm{g} / \mathrm{ml}$ of rt-PA for $60 \mathrm{~min}$ at $37^{\circ} \mathrm{C}$. At the end of the incubation period, the plasma levels of $B \beta 1-42$ were assayed as an index of fibrinogenolysis, whereas the extent of clot lysis was determined by counting the residual radioactivity of the clot. The amount of $B \beta 1-42$ generated was then expressed as a percent of the total releasable $B \beta 1-42$ which was calculated from the plasma fibrinogen concentration of $6.2 \mu \mathrm{M}$. Using linear regression analysis, the correlation coefficient is 0.9987 . 

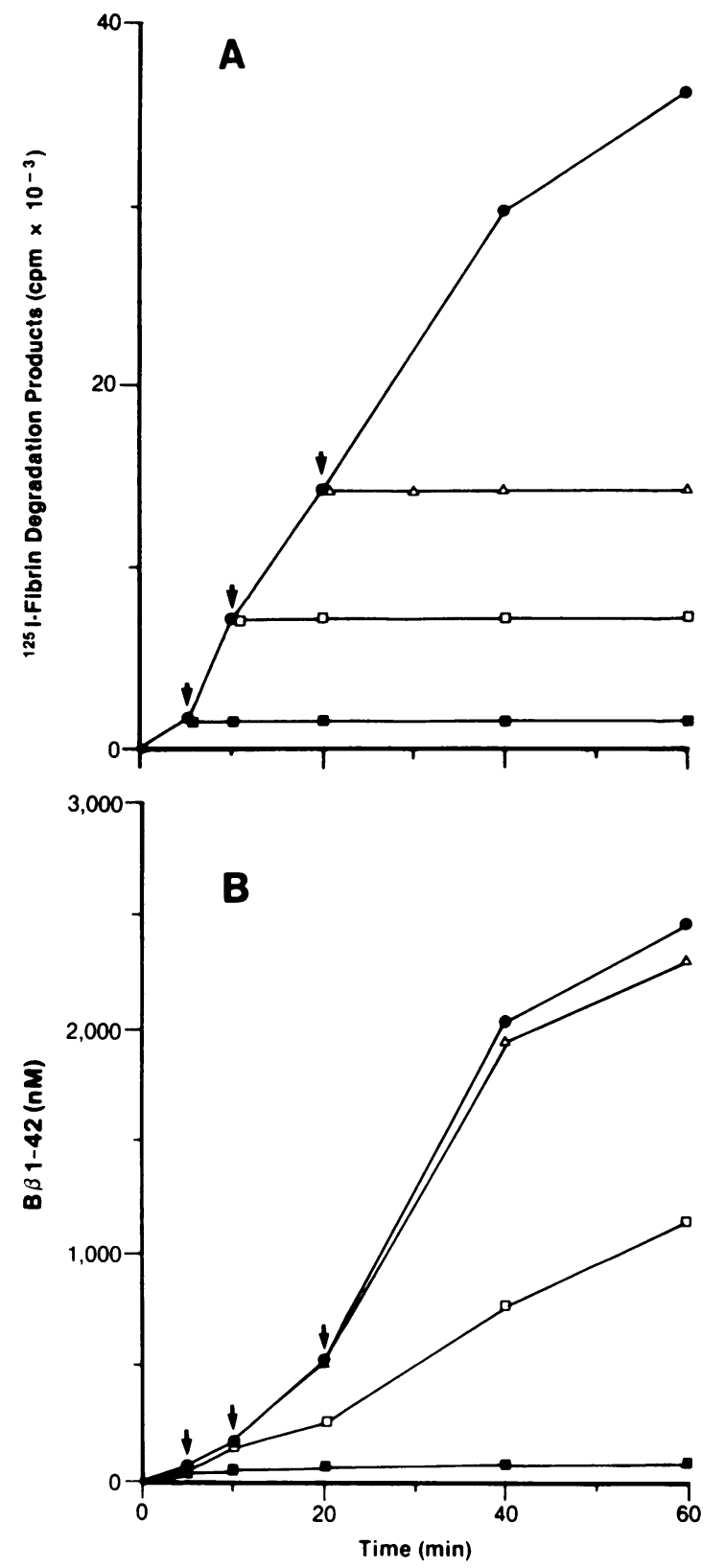

Figure 3. Time courses of concomitant rt-PA-induced release of fibrin degradation products from ${ }^{125}$ I-labeled fibrin clots $(A)$ and B $\beta 1-42$ generation $(B) .{ }^{125}$ I-labeled fibrin clots were incubated in plasma containing $1 \mu \mathrm{g} / \mathrm{ml}$ of rt-PA for $60 \mathrm{~min}$ at $37^{\circ} \mathrm{C}$. At the times indicated, aliquots were removed and the concentrations of ${ }^{125} \mathrm{I}$-labeled fibrin degradation products and $\mathrm{B} \beta 1-42$ were determined (๑). To examine the effect of early removal of the clot on clot lysis and fibrinogenolysis, ${ }^{125}$ I-labeled clots incubating in plasma containing 1 $\mu \mathrm{g} / \mathrm{ml}$ of $\mathrm{rt}-\mathrm{PA}$ were removed at the times indicated by the arrows, and the time courses of release of ${ }^{125} \mathrm{I}$-labeled fibrin degradation products and $B \beta 1-42$ generation were then determined. The effect of clot removal at $5 \mathrm{~min}(\square), 10 \mathrm{~min}(\square)$, or $20 \mathrm{~min}(\Delta)$ incubation is illustrated. Each point represents the mean of three separate experiments, each done in duplicate.

products (Fig. $3 A$ ), whereas fibrinogenolysis was followed by measuring the generation of $\mathrm{B} \beta 1-42$ (Fig. $3 \mathrm{~B}$ ). As indicated in Fig. $3 A$, the concentration of released ${ }^{125}$ I-labeled fibrin degradation products depends on the duration of exposure of the clots to rt-PA. Once the clots are removed from the rt-PA containing plasma, release of ${ }^{125} \mathrm{I}$-labeled fibrin degradation products ceases, whereas fibrinogenolysis continues (Fig. $3 \mathrm{~B}$ ). Thus, even though removal of the clots after 20 min incubation in rt-PA-containing plasma results in only $25 \%$ clot lysis, the time course of $\mathrm{B} \beta 1-42$ generation is almost identical to that which occurs when clots undergo $63 \%$ clot lysis by exposure to rt-PA for $60 \mathrm{~min}$. Similarly, there is ongoing B $\beta 1-42$ generation when clots are removed after $10 \mathrm{~min}$ incubation with rt-PA, although only $13 \%$ clot lysis has occurred by this time. In contrast, a 5-min exposure to rt-PA results in only $3 \%$ clot lysis, and there is little increase in the plasma levels of $B \beta 1-42$ if the clots are removed at this time. These findings indicate that fibrinogenolysis cannot be ascribed to plasmin produced on the clot surface because $B \beta 1-42$ generation continues after the clot is removed. Instead, the ongoing fibrinogen proteolysis is primarily mediated by soluble fibrin degradation products because once $25 \%$ lysis of the clot has been achieved by a 20 -min exposure to rt-PA, the amount of $\mathrm{B} \beta 1-42$ generated is almost the same as that produced when clots are incubated with rt-PA for $60 \mathrm{~min}$.

Potentiation of rt-PA-induced fibrinogenolysis by clot lysates. To determine whether soluble fibrin degradation products can potentiate rt-PA-mediated fibrinogenolysis, varying amounts of clot lysate were incubated with rt-PA-containing plasma, and fibrinogenolysis was monitored by measuring the levels of $B \beta 1-42$. As illustrated in Fig. 4, the addition of clot lysate produces a concentration-dependent increase in the amount of $\mathrm{B} \beta 1-42$ that is generated. These studies thus indicate that soluble fibrin degradation products can potentiate rt-PAinduced fibrinogenolysis.

Adsorption of ${ }^{125}$ I-labeled fibrin degradation products to plasminogen-Sepharose or rt-PA-Sepharose. To explore the mechanism by which soluble fibrin degradation products potentiate rt-PA-induced fibrinogenolysis, we set out to determine whether these derivatives can bind plasminogen and/or rt-PA by examining the adsorption of these products to plasminogen-Sepharose or rt-PA-Sepharose. $\sim 72 \%$ of the ${ }^{125} \mathrm{I}-\mathrm{la}$ beled fibrin degradation products in the crude clot lysates binds to both plasminogen-Sepharose and rt-PA-Sepharose (data not shown). The bound and unbound fractions were analyzed using SDS-PAGE followed by autoradiography (Fig. 5). Similar fibrin-derived degradation products bind to both plasminogenSepharose and to rt-PA-Sepharose. In each case, two major bands are visualized at $\sim 195,000$ and $60,000 \mathrm{D}$, respectively.

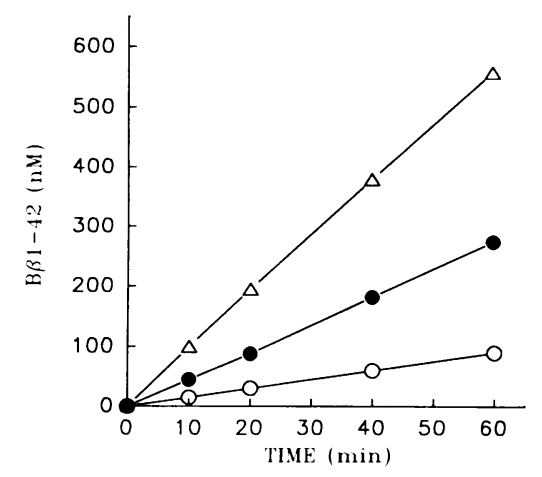

Figure 4. Potentiation of rt-PA-induced $\mathrm{B} \beta 1$. 42 generation by clot lysates. Plasma was incubated with $1 \mu \mathrm{g} / \mathrm{ml}$ of rt-PA for $60 \mathrm{~min}$ at $37^{\circ} \mathrm{C}$ in the absence (O) or presence of $2.5 \mu \mathrm{l}(\bullet)$ or $5 \mu \mathrm{l}(\Delta)$ of clot lysate. At the times indicated, aliquots were removed, unreacted fbrinogen was precipitated with ethanol, and the ethanol supernatants were assayed for $\mathrm{B} \beta 1-42$. 


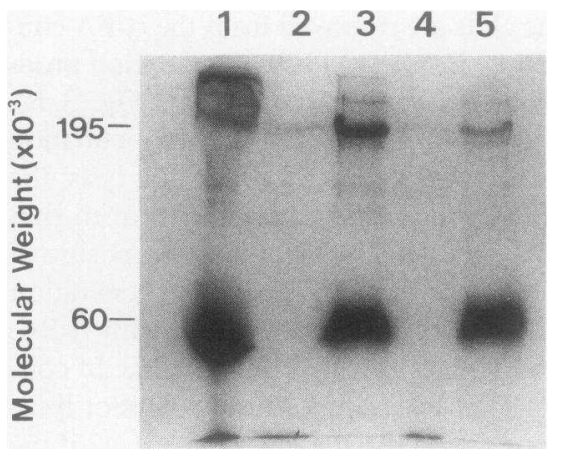

Figure 5. Autoradiographic analysis of clot lysates. The crude clot lysate is shown in lane 1 . Lanes 3 and 5 illustrate the fractions of lysate eluted from plasminogen-Sepharose and rt-PA-Sepharose, respectively, whereas lanes 2 and 4 show the lysate fractions that do not bind to these adsorbants.

In addition, higher molecular weight bands also are seen. SDSPAGE analysis of the material that does not bind to plasminogen-Sepharose or rt-PA-Sepharose shows only a faint band at 195,000 D.

Potentiating activity of bound and unbound fractions. The bound and unbound fractions also were tested for their ability to potentiate rt-PA-induced $\mathrm{B} \beta 1-42$ generation in plasma, and their potentiating activity was then compared with that of the crude lysate. Those fibrin degradation products that bind to plasminogen-Sepharose or to rt-PA-Sepharose potentiate $B \beta 1$ 42 generation to an extent similar to that produced by the crude lysates (Table II). In contrast, the unbound fractions

Table II. Potentiation of rt-PA-induced B $\beta 1-42$ Generation by Clot Lysates Before and After Adsorption with PlasminogenSepharose or rt-PA-Sepharose

\begin{tabular}{lcc}
\hline \multicolumn{1}{c}{ Adsorbant } & B $\beta 1-42$ & $\begin{array}{c}\text { Increase in } \\
\text { B } \beta 1-42\end{array}$ \\
& $n M$ & \\
None & 1346.5 & 14.7 \\
Plasminogen-Sepharose & 4.2 & $<1$ \\
t-PA-Sepharose & 1.2 & $<1$ \\
Plasminogen-Sepharose eluate & 1234.5 & 13.5 \\
t-PA-Sepharose eluate & 1263.2 & 13.8 \\
& &
\end{tabular}

rt-PA $(2 \mu \mathrm{g} / \mathrm{ml})$ was incubated in plasma for $60 \mathrm{~min}$ at $37^{\circ} \mathrm{C}$ in the presence or absence of $10 \mu \mathrm{l}$ of clot lysate. At the end of the incubation period, unreacted fibrinogen was precipitated with ethanol, and the ethanol supernatants were assayed for $B \beta 1-42$ as an index of fibrinogenolysis. The $\mathrm{B} \beta 1-42$ generated by rt-PA alone ( $91.6 \mathrm{nM})$ was subtracted so that the potentiating effect of the lysate could be calculated. This value divided by the basal $\mathrm{B} \beta 1-42$ generation of 91.6 $\mathrm{nM}$ represents the increase in $\mathrm{B} \beta-42$ produced by the lysate. Using the same assay system, the activity of the clot lysate was again determined after adsorption with plasminogen-Sepharose or t-PA-Sepharose. The ${ }^{125}$ I-labeled fibrin degradation products that bound to plasminogen-Sepharose or t-PA-Sepharose were then eluted as described in Methods, and the activity of $10 \mu \mathrm{l}$ of each of the eluates was measured. The results shown represent the mean of two separate experiments, each done in duplicate. have virtually no potentiating activity. When the crude clot lysates or the fractions that bound to plasminogen-Sepharose or rt-PA-Sepharose were concentrated by lyophylization rather than by ultrafiltration, their potentiating activity was lost, suggesting that the three-dimensional structure of these fragments is an important determinant of their activity.

Gel filtration of fibrin degradation products that bind to plasminogen-Sepharose and to rt-PA-Sepharose. To further characterize the fibrin degradation products that potentiate rtPA-induced fibrinogenolysis, the crude clot lysates and those fractions of the lysates that bound to plasminogen-Sepharose or to rt-PA-Sepharose were subjected to chromatography on Sephacryl S300 HR to separate the products according to their molecular weights. As illustrated in Fig. 6, gel filtration of the fractions of clot lysate that bound to plasminogen-Sepharose identified a major protein peak (peak B) which based on its elution profile, has a molecular weight of $\sim 255,000 \mathrm{D}$. In addition, a small high-molecular weight peak (peak $A$ ) that eluted in the void volume, and a minor peak (peak C) of molecular weight $\sim 60,000 \mathrm{D}$ also were identified. Chromatography of the crude lysate and the fractions of the lysate that bound to rt-PA-Sepharose revealed similar peaks (data not shown). The fractions from the gel filtration column were collected, pooled as indicated, concentrated by ultrafiltration, and then characterized by PAGE and immunoblot analysis, and tested for their ability to potentiate rt-PA-induced fibrinogenolysis in plasma.

Characterization of fibrin degradation products. The gel filtration fractions were characterized using a combination of PAGE and immunoblot analysis. Electrophoresis was performed under both dissociating (Fig. 7) and nondissociating conditions (Fig. 8). The main protein fraction, peak B (Fig. 6), was identified as consisting primarily of (DD)E complex because under nondissociating conditions (Fig. 8) there is a single 255,000-D band (Fig. $8 A$, lane 3 ) that is recognized both by the antibody against DD (Fig. $8 \mathrm{~B}$, lane 3 ) and by the antibody against fragment $\mathrm{E}$ (Fig. $8 \mathrm{C}$, lane 3 ). In contrast, in the presence of SDS (Fig. 7) this material separates into two bands at $\sim$ 195,000 and 60,000 D, respectively (Fig. $7 A$, lane 3 ). The higher molecular weight band is recognized by a monoclonal antibody against DD (Fig. $7 \mathrm{~B}$, lane 3 ), whereas the lower molecular weight band reacts with an antibody against fragment $\mathrm{E}$ (Fig. $7 C$, lane 3).

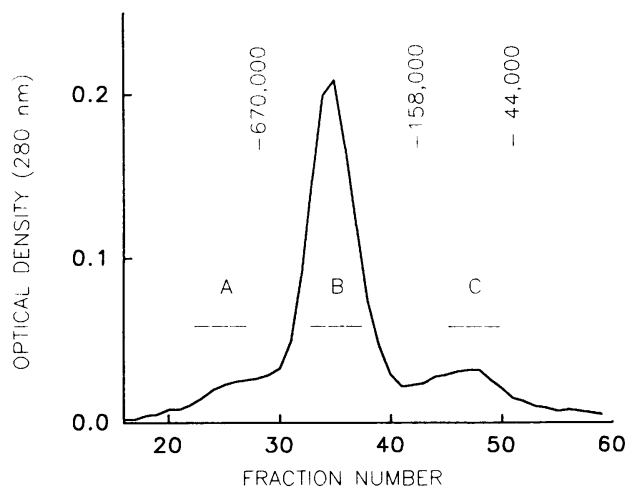

Figure 6. Gel filtration profile of clot lysate separated on a Sephacryl S300R column. The elution positions of standard proteins with known molecular weights are shown by the vertical lines. 2-ml fractions were collected, and pooled as indicated. 


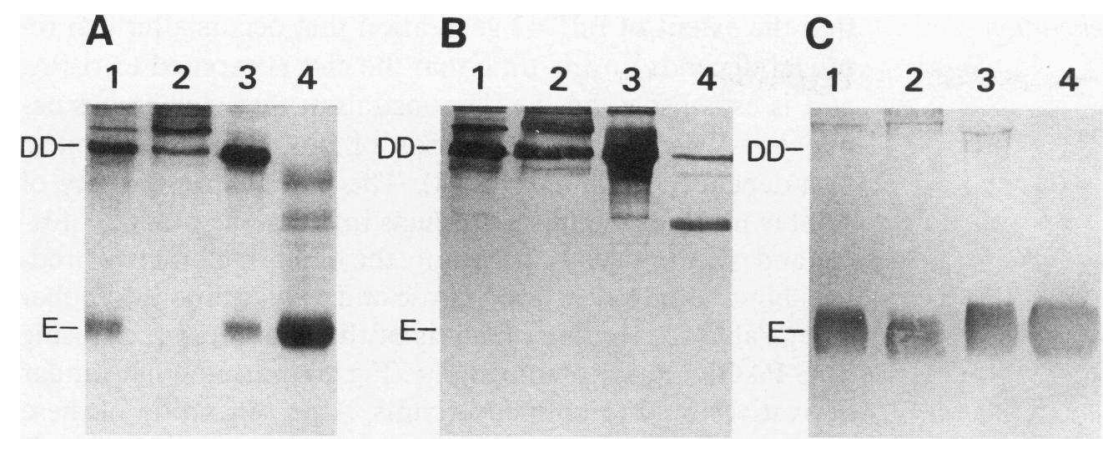

Figure 7. SDS-PAGE and immunoblot analysis of the degradation products of cross-linked fibrin. In $A$, the gels were stained with Coomasie blue. The separated proteins also were electrophoretically transferred to nitrocellulose membranes, which were then reacted with a monoclonal antibody against DD $(B)$ or a monospecific antibody against fragment $\mathrm{E}(C)$. Lane $l$ shows the crude lysate, whereas lanes 2-4 represent the gel filtration peaks $\mathrm{A}, \mathrm{B}$, and $\mathrm{C}$, respectively, that are shown in Fig. 6.

PAGE analysis under dissociating conditions (Fig. $7 \mathrm{~A}$, lane 2) of the peak that elutes in the void volume of the gel filtration column (Fig. 6, peak A) reveals several high-molecular weight bands. In addition, there are higher molecular weight derivatives that do not enter the $4 \%$ stacking gel. Those fragments that enter the gel are recognized by the antibody against DD (Fig. $7 B$, lane 2). Although not visualized on the gel stained with Coomasie blue (Fig. $7 A$, lane 2), these derivatives also contain material that migrates at $\sim 60,000 \mathrm{D}$ and is recognized by the antibody against fragment $\mathrm{E}$ (Fig. $7 C$, lane 2). Under dissociating conditions a band of molecular weight greater than the (DD)E complex is visualized (Fig. $8 A$, lane 2 ). This band is recognized both by an antibody against DD (Fig. $8 \mathrm{~B}$, lane 2 ) and by an antibody against fragment $\mathrm{E}$ (Fig. $8 C$, lane 2 ). Thus, these species represent high molecular weight derivatives that contain the (DD)E complex.

The lowest molecular weight peak recovered from the gel filtration column (Fig. 6, peak C) migrates as a major band of $\sim 60,000 \mathrm{D}$ under dissociating conditions (Fig. $7 \mathrm{~A}$, lane 4 ) that is recognized by the antibody against fragment $\mathrm{E}$ (Fig. $7 \mathrm{C}$, lane 4 ) but not by the antibody against DD (Fig. $7 B$, lane 4 ). In addition, at least two higher molecular weight bands that are recognized by the antibody against DD also are visualized (Fig. $7 \mathrm{~B}$, lane 4 ). These bands are of lower molecular weight than intact $\mathrm{DD}$, and probably represent products of its digestion by plasmin. Under nondissociating conditions, peak $\mathrm{C}$ migrates as a minor band at $\sim 60,000 \mathrm{D}$ and a major band at $\sim 55,000 \mathrm{D}$ (Fig. $8 \mathrm{~A}$, lane 4 ). These bands are recognized by the antibody against fragment $\mathrm{E}$ (Fig. $8 \mathrm{C}$, lane 4 ) and not by the antibody against DD (Fig. $8 \mathrm{~B}$, lane 4 ), indicating that they represent fragment $E$ species.

The fibrin degradation products that do not bind to plasminogen-Sepharose or rt-PA-Sepharose (Fig. 5, lanes 2 and 4, respectively) also were characterized by SDS-PAGE and immunoblot analysis (data not shown). This material consists primarily of DD because the 195,000-D band visualized in Fig. 5 is recognized by an antibody against DD. Little or no fragment $E$ is present because a 60,000 -D band is not seen in Fig. 5 nor is a distinct band visualized on immunoblot analysis using the antibody against fragment $\mathrm{E}$.

Potentiation of rt-PA-induced fibrinogenolysis by isolated degradation products of cross-linked fibrin. The fractions representing peaks $\mathrm{A}, \mathrm{B}$, and $\mathrm{C}$ were tested for their ability to potentiate rt-PA-induced $\mathrm{B} \beta 1-42$ generation in plasma, and their activity was compared with that of the starting material (Table III). All of the plasmic degradation products of cross-linked fibrin that bind to plasminogen-Sepharose or to rt-PA-Sepharose have the potential to augment rt-PA-induced fibrinogenolysis and over $75 \%$ of the activity of the starting material is recovered (data not shown). On a gravimetric basis, however, (DD)E complex is the most potent stimulator (Table III). This finding may explain the substantial fibrinogenolysis that accompanies rt-PA-induced clot lysis because (DD)E complex is the major derivative found in the clot lysates. In contrast, DD, which is the predominant species in those fractions that do not bind to plasminogen-Sepharose or to rt-PA-Sepharose (Fig. 5) does not have potentiating activity (Table II).

\section{Discussion}

The activation of plasminogen by t-PA is accelerated in the presence of fibrin $(7,8)$. Although fibrinogen has little effect on the rate of t-PA-induced plasminogen activation $(7,8)$, fragments of fibrinogen or fibrin can mimic the accelerating effect of intact fibrin (22-28). Rate-enhancing fragments can be generated by digesting fibrin(ogen) with plasmin or with cyanogen

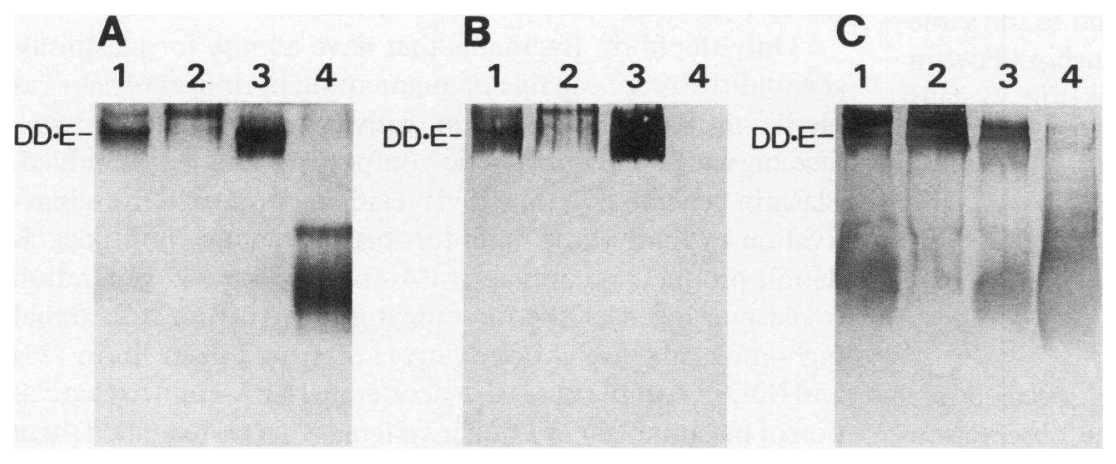

Figure 8. PAGE and immunoblot analysis of the degradation products of cross-linked fibrin done under nondissociating conditions. In panel $A$, the gels were stained with Coomasie blue. The separated proteins also were electrophoretically transferred to nitrocellulose membranes which were then reacted with a monoclonal antibody against $\mathrm{DD}(B)$ or a monospecific antibody against fragment $\mathrm{E}(C)$. Lane $l$ shows the crude lysate, whereas lanes 2-4 represent the gel filtration peaks $A, B$, and $C$, respectively, that are shown in Fig. 6. 
Table III. Potentiation of rt-PA-induced B B1-42 Generation by Isolated Fibrin Degradation Products

\begin{tabular}{lc}
\hline \multicolumn{1}{c}{ Species } & $\begin{array}{c}\text { Increase in } \mathrm{B} \beta 1-42 \text { for } \\
\text { each } \mu \mathrm{g} \text { protein }\end{array}$ \\
\hline Plasminogen-Sepharose eluate & $n M$ \\
High-molecular weight derivatives & $206 \pm 32$ \\
(DD)E Complex & $18 \pm 5$ \\
Fragment E & $110 \pm 28$ \\
& $32 \pm 8$ \\
\hline
\end{tabular}

The potentiating activity of the fraction of the crude clot lysates that bound to plasminogen-Sepharose was determined by incubating four different concentrations of this material with plasma for $60 \mathrm{~min}$ at $37^{\circ} \mathrm{C}$ in the presence or absence of $1 \mu \mathrm{g} / \mathrm{ml}$ of rt-PA. At the end of the incubation period, unreacted fibrinogen was precipitated with ethanol, and the ethanol supernatants were assayed for $B \beta 1-42$. The increase in rt-PA-induced generation of $\mathrm{B} \beta 1-42$ produced by the plasminogen-Sepharose eluate was then calculated. The eluate was then subjected to chromatography on Sephacryl S300HR as described in Methods, and the fractions pooled as indicated in Fig. 6 . The potentiating activity of peaks $\mathrm{A}, \mathrm{B}$, and $\mathrm{C}$ which represent high-molecular weight derivatives that contain the (DD)E complex, (DD)E complex itself, and fragment $\mathrm{E}$, respectively, was then tested as described above. The values illustrated represent the mean $\pm \mathrm{SD}$ of two different experiments each done in duplicate.

bromide. The physiologic significance of these observations is uncertain however, because many of these fragments are not produced in vivo. In addition, the studies reported to date have only examined the accelerating effect of fibrin(ogen) fragments in buffer systems, and the potential modulating effects of antiproteinases have not been considered. In the present study we have extended the observations made in buffer systems by $(a)$ demonstrating that the physiologic products of rt-PA-induced lysis of cross-linked fibrin can potentiate rt-PA-mediated fibrinogenolysis in a plasma system, $(b)$ identifying and characterizing those fibrin degradation products that have potentiating activity, and $(c)$ demonstrating that the products with potentiating activity bind both plasminogen and rt-PA, which suggests that they act by providing a surface on which the fibrinolytic components can be assembled.

In the presence of fibrin there is an $\sim 21$-fold increase in the amount of $B \beta 1-42$ generated by a wide range of rt-PA concentrations ( Table I). That the higher $B \beta 1-42$ concentrations in the presence of fibrin reflect increased fibrinogen proteolysis is confirmed by the results of immunoblot analysis which permits direct visualization of the fibrinogen degradation products (Fig. 1). When clots of increasing size are exposed to the same concentration of rt-PA, there is a linear relationship between the extent of clot lysis and that of fibrinogenolysis (Fig. 2). This phenomenon cannot be explained on the basis of the larger clots providing a greater surface on which plasmin can be generated because there is ongoing $B \beta 1-42$ generation after the clots are removed from rt-PA-containing plasma (Fig. 3). If fibrinogenolysis was mediated by plasmin bound to the clot surface, $B \beta 1-42$ production would cease when the clot is removed.

That soluble fibrin degradation products are responsible for the ongoing fibrinogenolysis is suggested by the observation that the extent of $\mathrm{B} \beta 1-42$ generation that occurs after clot removal depends on the time that the clot is exposed to rt-PA, and is established by the demonstration that clot lysates enhance rt-PA-mediated generation of $B \beta 1-42$ in a concentration-dependent fashion (Fig. 4). The potentiating activity of soluble fibrin degradation products involves the binding of rtPA and plasminogen because only the functionally active products bind both to rt-PA-Sepharose and to plasminogen-Sepharose (Table II). Further, analysis of the bound products using SDS-PAGE and autoradiography (Fig. 5) indicates that similar derivatives bind to both adsorbants. After separation of these products by gel filtration (Fig. 6), the predominant 255,000-D species (peak B) was identified as (DD)E complex by PAGE and immunoblot analysis (Figs. 7 and 8 ) consistent with previous reports that this complex is the major product of plasmin degradation of cross-linked fibrin (38-42). The minor peaks identified by gel filtration include a series of highmolecular weight species (peak A) that elutes in the void volume of the column, some of which have a mol wt $>10^{6}$ and do not enter the $4 \%$ stacking gel. Those products that enter the gel contain epitopes that are recognized both by an antibody against D-dimer (Fig. $7 \mathrm{~B}$ and $8 \mathrm{~B}$, lane 2 ) and by antibody against fragment $\mathrm{E}$ (Fig. $7 C$ and $8 C$, lane 2). Thus, these large fragments contain the (DD)E complex and represent the highmolecular weight derivatives that have been extensively characterized by Francis et al. $(31,43)$. The other minor peak identified by gel filtration (peak $C$ ) has a molecular weight of $\sim 60,000 \mathrm{D}$ and represents predominantly fragment $\mathrm{E}$. Analysis under nondissociating conditions indicates that more than one fragment $\mathrm{E}$ species is present (Fig. $8 \mathrm{~A}$, lane 4 ). These findings are consistent with those of Olexa and Budzynski (42, $44,45)$ who identified three major fragment $E$ species that were designated E1, E2, and E3 according to their increasing electrophoretic mobility. These three fragment $E$ derivatives represent sequential plasmic degradation products, and only E1 and E2 are capable of binding to DD $(44,45)$.

Whereas high-molecular weight derivatives that contain the (DD)E complex, (DD)E complex itself, and fragment $E$ bind to both plasminogen-Sepharose and rt-PA-Sepharose, DD does not bind (Fig. 5). These findings suggest that a binding site for both plasminogen and rt-PA is located within the E domain. Although the binding sites for t-PA have not previously been defined, plasminogen binding sites have been identified on the amino-terminal regions of the $\mathrm{A} \alpha$ - and $\mathrm{B} \beta$ chains of fragment $E$ (46-48). Fibrinogen fragment $D$ also contains plasminogen binding sites $(47,49)$. The finding that DD does not bind to plasminogen-Sepharose suggests that these sites are inaccessible when gamma-gamma cross-linking of adjacent $\mathrm{D}$ domains results in DD formation.

Only the fibrin fragments that have affinity for plasminogen and rt-PA are capable of augmenting fibrinogenolysis (Table II) suggesting that these derivatives act by providing a surface on which the fibrinolytic components can be assembled. Plasmin generated at this site is relatively protected from inactivation by fluid-phase inhibitors because soluble fibrin degradation products potentiate rt-PA-induced $\mathrm{B} \beta 1-42$ generation in plasma (Fig. 4). Other investigators have demonstrated that high-molecular weight derivatives of cross-linked fibrin (27) and (DD)E complex (28) can accelerate rt-PA-induced activation of plasminogen in a buffer system. We have extended these 
observations in three ways. First, we have shown that in addition to these derivatives, fragment $\mathrm{E}$ also potentiates rt-PA-induced fibrinogenolysis (Table III). Quantitatively, however, (DD)E complex, the major product of plasmin degradation of cross-linked fibrin, is the most potent stimulator of fibrinogenolysis (Table III). Second, because our studies were done in a plasma system, they indicate that plasmin generated on the surface of fibrin degradation products is relatively protected from inactivation by fluid-phase antiplasmins. Third, the data presented in Fig. 3 suggest that plasmin generated on the surface of fibrin degradation products is a more important mediator of fibrinogenolysis than plasmin generated on the clot surface.

In summary, we have demonstrated that the products of rt-PA-induced degradation of cross-linked fibrin can potentiate rt-PA-mediated fibrinogenolysis by providing a surface for t-PA and plasminogen binding thereby promoting plasmin generation. The occurrence of this phenomenon after therapeutic thrombolysis may explain the limited fibrin selectivity of rt-PA.

\section{Acknowledgments}

The authors wish to thank Dr. J. Hirsh and Dr. A. Budzynski for their excellent advice and encouragement, and S. Crnic for preparing this manuscript.

This work was supported by grants from the Heart and Stroke Foundation of Ontario and the Medical Research Council of Canada. Dr. Weitz is a Scholar of the Heart and Stroke Foundation of Ontario.

\section{References}

1. Wiman, B., and D. Collen. 1978. Molecular mechanisms of physiologic fibrinolysis. Nature (Lond.). 272:549-550.

2. Lucas, M. A., L. J. Fretto, and P. A. McKee. 1983. The binding of human plasminogen to fibrin and fibrinogen. J. Biol. Chem. 258:4249-4256.

3. Rakoczi, I., B. Wiman, and D. Collen. 1978. On the biological significance of the specific interaction between fibrin, plasminogen and antiplasmin. Biochim. Biophys. Acta. 540:295-300.

4. Rijken, D. C., M. Hoylaerts, and D. Collen. 1982. Fibrinolytic properties of one-chain and two-chain human extrinsic (tissue-type) plasminogen activators. $J$. Biol. Chem. 257:2920-2925.

5. Loscalzo, J. 1988. Structural and kinetic differences between single-chain and two-chain tissue plasminogen activator. J. Clin. Invest. 82:1391-1397.

6. Banyai, L., and L. Patthy. 1984. Importance of intramolecular interactions in the control of the fibrin affinity and activation of human plasminogen. J. Biol. Chem. 259:6466-6471.

7. Ranby, M. 1982. Studies on the kinetics of plasminogen activation by tissue plasminogen activator. Biochim. Biophys. Acta. 704:461-469.

8. Hoylaerts, M., D. C. Rijken, H. R. Lijnen, and D. Collen. 1982. Kinetics of the activation of plasminogen by human tissue plasminogen activator. Role of fibrin. J. Biol. Chem. 257:2912-2919.

9. Pennica, D., W. E. Holmes, W. J. Kohr, R. N. Harkins, G. A. Vehar, D. A. Ward, W. F. Bennett, E. Yelverton, P. H. Seeburg, H. L. Heyneker, D. V. Goeddel, and D. Collen. 1983. Cloning and expression of human tissue-type plasminogen activator cDNA in E. coli. Nature (Lond.). 301:214-221.

10. Topol, E. J., W. R. Bell, and M. L. Weisfeldt. 1985. Coronary thrombolysis with recombinant tissue-type plasminogen activator. A hematologic and pharmacologic study. Ann. Intern. Med. 103:837-843.

11. Van de Werf, F., P. A. Ludbrook, S. R. Bergmann, A. J. Tiefenbrunn, K. A. Fox, H. de Geest, M. Verstraete, D. Collen, and B. E. Sobel. 1984. Coronary thrombolysis with tissue-type plasminogen activator in patients with evolving myocardial infarction. N. Engl. J. Med. 310:609-613.

12. TIMI Study Group. 1985. The thrombolysis in myocardial infarction (TIMI) trial: phase 1 findings. $N$. Engl. J. Med. 312:932-936.

13. Rao, A. K., C. Pratt, A. Berke, A. Jaffe, I. Ockene, T. L. Schreiber, W. R. Bell, and M. Terrin. 1988. Thrombolysis in myocardial infarction (TIMI) trialphase 1: hemorrhagic manifestations and changes in plasma fibrinogen and the fibrinolytic system in patients treated with recombinant tissue plasminogen activator and streptokinase. J. Am. Coll. Cardiol. 11:1-11.

14. Verstraete, M., M. Bory, D. Collen, R. Erbel, R. J. Lennane, D. G. Mathey, H. R. Michels, M. Schartl, R. Uebis, R. Bernard, R. W. Brower, D. P. deBono, W. Huhmann, J. Lubsen, J. Meyer, W. Rutsch, W. Schmidt, and R. von Essen. 1985. Randomized trial of intravenous recombinant tissue type plasminogen activator versus intravenous streptokinase in acute myocardial infarction Lancet. $i: 842-847$.

15. Owen, J., K. D. Friedman, B. A. Grossman, C. Wilkins, A. D. Berke, and E. R. Powers. 1987. Quantitation of fragment $X$ formation during thrombolytic therapy with streptokinase or tissue plasminogen activator. J. Clin. Invest. 79:1642-1647.

16. Goldhaber, S. Z., D. E. Vaughan, J. E. Markis, A. P. Selwyn, M. E Meyerovitz, J. Loscalzo, D. S. Kim, C. M. Kessler, D. L. Dawley, G. V. R. K Sharma, A. Sasahara, E. B. Grossbard, and E. Braunwald. 1986. Acute pulmonary embolism treated with tissue plasminogen activator. Lancet. ii:886-889.

17. Verstraete, M., G. A. Miller, H. Bounameaux, B. Charbonnier, J. P. Colle, G. Lecorf, G. A. Marbet, P. Mombaerts, and C. G. Olsson. 1988. Intravenous and intrapulmonary recombinant tissue-type plasminogen activator in the treatment of acute massive pulmonary embolism. Circulation. 77:353-360.

18. Levine, M. N., J. Hirsh, J. Weitz, M. Cruickshank, J. Neemeh, A. G. G. Turpie, M. Andrew, M. Klimek, and M. Gent. 1990. A randomized trial of a single bolus dosage regimen of recombinant tissue plasminogen activator in patients with acute pulmonary embolism. Chest. 98:1473-1479.

19. Turpie, A. G. G. 1987. Thrombolytic therapy in venous thromboeombolism. In Tissue Plasminogen Activator in Thrombolytic Therapy. B. E. Sobel, D. Collen, and E. B. Grossbard, editors. Marcel Dekker, Inc., New York. 131-146.

20. Sobel, B. E., R. W. Gross, and A. K. Robison. 1984. Thrombolysis, clot selectivity, and kinetics. Circulation. 70:160-164.

21. Noe, D. A., and W. R. Bell. 1987. A kinetic analysis of fibrinogenolysis during plasminogen activator therapy. Clin. Pharmacol. Ther. 41:297-303.

22. Nieuwenhuizen, W., A. Vermond, M. Voskuilen, D. W. Traas, and J. H. Verheijen. 1983. Identification of a site in fibrin(ogen) which is involved in the acceleration of plasminogen activation by tissue-type plasminogen activator. Biochim. Biophys. Acta. 748:86-92.

23. Verheijen, J. H., W. Nieuwenhuizen, and G. Wijngaards. 1982. Activation of plasminogen by tissue activator is increased specifically in the presence of certain soluble fibrin(ogen) fragments. Thromb. Res. 27:377-385.

24. Verheijen, J. H., W. Neiuwenhuizen, D. W. Traas, G. T. G. Chang, and E. Hoegee. 1983. Differences in effects of fibrin(ogen) fragments on the activation of 1-glu-plasminogen and 442-val-plasminogen by tissue-type plasminogen activator. Thromb. Res. 32:87-92.

25. Nieuwenhuizen, W., J. H. Verheijen, A. Vermond, G. T. G. Chang. 1983 Plasminogen activation by tissue activator is accelerated in the presence of $\mathrm{fi}$ brin(ogen) cyanogen bromide fragment FCB-2. Biochim. Biophys. Acta. 755:531-533.

26. Voskuilen, M., A. Vermond, G. H. Veeneman, J. H. Van Boom, E. A. Klasen, N. D. Zegers, and W. Neiuwenhuizen. 1987. Fibrinogen lysine residue A $\alpha 157$ plays a crucial role in the fibrin-induced acceleration of plasminogen activation catalyzed by tissue-type plasminogen activator. J. Biol. Chem. 262:5944-5946

27. Wilhelm, O. G., and N. U. Bang. 1988. The influence of diverse fibrins and fibrin(ogen) fragments on the acceleration of tissue-plasminogen activatormediated plasminogen activation. In Fibrinogen 3. Biochemistry, Biologic Functions, Gene Regulation and Expression. M. W. Mossesson, D. L. Amrani, K. R. Siebenlist, J. P. Diorio, editors. Elsevier Science Publishers, Amsterdam. 181184.

28. Hasan, A. K., and A. Z. Budzynski. 1989. Binding of one-chain and two-chain t-PA to fibrin fragments. Circulation. 80:2552a. (Abstr.)

29. Ikeno, L. C., B. M. Bowen, and M. Der. 1981. Commercial production of 125I-fibrinogen injection. J. Radioanal. Chem. 65:179-188.

30. McFarlane, A. S. 1965. Labelling of plasma proteins with radioactive iodine. Biochem. J. 62:135-143.

31. Francis, C. W., V. J. Marder, and S. E. Martin. 1980. Plasmic degradation of crosslinked fibrin. I. Structural analysis of the particulate clot and identification of new macromolecular-soluble complexes. Blood. 56:456-464.

32. Weitz, J. I., J. A. Koehn, R. E. Canfield, S. L. Landman, and R. Friedman. 1986. Development of a radioimmunoassay for the fibrinogen-derived fragment B $\beta 1$-42. Blood. 67:1014-1022.

33. MacGregor, I. R., L. R. Micklem, K. James, and D. S. Pepper. 1985. Characterization of epitopes on human tissue plasminogen activator recognized by a group of monoclonal antibodies. Thromb. Haemostasis. 53:45-50.

34. Lowry, O. H., N. J. Rosebrough, A. L. Farr, and R. J. Randall. 1951. Protein measurement with the folin phenol reagent. J. Biol. Chem. 193:265-275.

35. Laemmli, U. K. 1970. Cleavage of structural proteins during the assembly of the head of bacteriophage T4. Nature (Lond.). 227:680-685.

36. Davis, B. J. 1964. Disc electrophoresis. II. Method and application to human serum proteins. Ann. NY Acad. Sci. 121:404-427.

37. Garabedian, H. D., H. K. Gold, R. C. Leinbach, J. A. Johns, T. Yasuda, 
M. Kanke, and D. Collen. 1987. Comparative properties of two clinical preparations of recombinant human tissue-type plasminogen activator in patients with acute myocardial infarction. J. Am. Coll. Cardiol. 9:599-607.

38. Gaffney, P. J., and M. Brasher. 1973. Subunit structure of the plasmin-induced degradation products of crosslinked fibrin. Biochim. Biophys. Acta. 295:308-313.

39. Hudry-Clergeon, G., L. Paturel, and M. Suscillon. 1974. Identification d'un complexe (D-D) . . . E dans les produits de degradation de la fibrine bovine stabilisee par le facteur XIII. Pathol. Biol. 22(Suppl.):47-52.

40. Kopec, M., E. Teisseyre, G. Dudek-Wojciechowska, M. Koczewiak, A Pankiewicz, and Z. S. Latallo. 1973. Studies on the "Double D" fragment from stabilized bovine fibrin. Thromb. Res. 2:283-291.

41. Pizzo, S. V., L. M. Taylor, Jr., M. L. Schwartz, R. L. Hill, and P. A

McKee. 1973. Subunit structure of fragment $D$ from fibrinogen and crosslinked fibrin. J. Biol. Chem. 248:4584-4590.

42. Olexa, S. A., and A. Z. Budzynski. 1979. Primary soluble plasmic degradation product of human cross-linked fibrin. Isolation and stoichiometry of the (DD)E complex. Biochemistry. 18:991-995.

43. Francis, C. W., V. J. Marder, and G. H. Barlow. 1980. Plasmic degrada- tion of crosslinked fibrin. Characterization of new macromolecular soluble complexes and a model of their structure. J. Clin. Invest. 66:1033-1043.

44. Olexa, S. A., and A. Z. Budzynski. 1979. Binding phenomena of isolated unique plasmic degradation products of human cross-linked fibrin. J. Biol. Chem. 254:4925-4932.

45. Olexa, S. A., A. Z. Budzynski, R. F. Doolittle, B. A. Cottrell, and T. C. Greene. 1981. Structure of fragment E species from human cross-linked fibrin. Biochemistry. 20:6139-6145.

46. Cederholm-Williams, S. A., and S. J. Fennel. 1981. Binding of plasmin(ogen) to sepharose bound fibrin(ogen) alpha-chain. Thromb. Res. 21:503506.

47. Varadi, A., and L. Patthy. 1983. Location of plasminogen-binding sites in human fibrin(ogen). Biochemistry. 22:2440-2446.

48. Varadi, A., and L. Patthy. 1984. Beta $\left(\operatorname{Leu}_{121}-\mathrm{Lys}_{122}\right)$ segment of fibrinogen is in a region essential for plasminogen binding by fibrin fragment $\mathrm{E}$. Biochem istry. 23:2108-2112.

49. Christensen, U. 1985. C-Terminal lysine residues of fibrinogen fragments essential for binding to plasminogen. FEBS (Fed. Eur. Biochem. Soc.) Lett 182:43-46. 